\title{
Schizophrenic and paranoid thinking in conceptual performance
}

\author{
GREG B. SIMPSON \\ University of Kansas, Lawrence, Kansas 66045 \\ LYLE E. BOURNE, JR. \\ University of Colorado, Boulder, Colorado 80302 \\ DON R. JUSTESEN and ROBERT J. RHODES \\ University of Kansas School of Medicine, Kansas City, Kansas 66103
}

\begin{abstract}
Thirty-two hospitalized patients diagnosed as schizophrenic were assigned in equal numbers to four groups on the basis of their scores on the Whitaker Index of Schizophrenic Thinking (WIST) and the paranoid (Pa) scale of the MMPI. Subjects attempted to solve two conceptformation problems in which they were to identify relevant stimulus attributes, under two levels of demand on their memories for previous stimuli. Only in conjunction with a low score on the $\mathrm{Pa}$ scale did the schizophrenic index predict a conceptual deficit. Only the highWIST, low-Pa group differed significantly from a control group of normal subjects. This result qualifies earlier research concerning the power of the WIST to predict logical performance. No difference was found between the two levels of memory demand.
\end{abstract}

Conceptual behavior may be broadly defined as the collection of skills and knowledge that allows people to recognize regularities among objects and events, and to use those regularities to organize their cognitive representations of the world. Recent research (Bourne, Justesen, Abraham, Beeker, Brauchi, Whitaker, \& Yaroush, 1977) has shown that the conceptual skills of a sample of patients diagnosed as schizophrenic varied as a function of the severity of schizophrenic thinking, as measured by the Whitaker Index of Schizophrenic Thinking (WIST) (Whitaker, 1973).

The WIST is a short (25-item) objective test that measures the illogicality of subjects' associations to single words, word pairs, and sentences that describe hypothetical inventions. Higher WIST indices indicate greater disturbance of thinking. In general, those patients who made unusual associations to the verbal stimuli on the WIST alsu failed to understand and abstract the relationship among visual stimuli required by a conceptual rule-learning task. Conspicuous,

This research was supported in part by HEW Research Service Award HD 07066 from the National Institute of Child Health and Human Development to the Kansas Center for Mental Retardation and Human Development. It was also supported in part by research Grant MH-14314 and by Research Scientist Award 1-K5-MH37497 from the National Institute of Mental Health to the second author, and by research Grant BNS76-81416 from the National Science Foundation to the second author. We are grateful to E. L. Wike for his technical advice and for reading an earlier version, and to the staff and residents of Osawatomie State Hospital for their cooperation and assistance. Requests for reprints should be sent to Greg Simpson, Department of Psychology, University of Kansas, Lawrence, Kansas 66045. however, was the normal conceptual performance of several patients with high WIST indices who, by psychiatric diagnosis, were also paranoid (Beeker, 1974).

That the paranoid schizophrenic subject may be less deficient in conceptual skills than the nonparanoid is consistent with conclusions drawn from studies of other cognitive abilities. Thinking in nonparanoid subjects has been reported as more concrete and regressive than in paranoid subjects (Wahl \& Wishner, 1972; Youkilis \& De Wolfe, 1975), and Wahl \& Wishner (1972) have suggested that there are basic differences in the thinking disorders of the two groups. Silverman (1964) has reviewed research showing that schizophrenic subjects with paranoid tendencies are more proficient than nonparanoid subjects in attending selectively to features of a complex visual stimulus, and also show more extensive and repeated scanning of a visual display.

Complex conceptual behavior, however, consists of much more than simple selective attention to stimuli. It includes the identification of those stimulus attributes that are critical to understanding a concept, and the mastery of a logical rule that describes how these attributes are related. The purpose of the study reported here was to determine whether one of these conceptual skills, the identification of critical stimulus attributes, differs among chronic schizophrenic patients when there is an overlay of paranoid thinking. Consistent with the results of Bourne et al. (1977), it was expected that subjects scoring low on the WIST would have little difficulty with attribute-identification problems. Those scoring higher on the WIST should have greater difficulty with the problems in general, but it is at 
this level that we also expected to see a difference between paranoid and nonparanoid subjects. In light of the better attentional and perceptual skills found in paranoid subjects, we would expect that this highWIST group would also perform better on abstract conceptual problems. Such a difference would not be expected for the low-WIST group, as the lower index indicates that the subject's logical abilities are relatively unimpaired at the time of testing.

Performance was also assessed under two levels of demand on the subjects' memories for recently presented stimuli. Bourne, Goldstein, and Link (1964) showed that, for normal subjects, solution of conceptual problems was more rapid when stimuli of previous trials remained in view (termed "external" memory) than when they were removed after each trial. It was expected that the paranoid subjects would be better able than nonparanoid to use the extra information provided by an external memory condition.

\section{METHOD}

\section{Subjects}

Thirty-two patients at Osawatomie State Hospital (Kansas), diagnosed as schizophrenic, served as subjects. All were between 20 and 40 years of age and were in their third, fourth, or fifth hospitalization. None had a record of central nervous system damage or gross sensory defect. All were receiving daily dosages of phenothiazines. Eight normal control subjects were students in general psychology classes at the Universities of Missouri (at Kansas City) and Kansas, between the ages of 18 and 55 years.

\section{Stimuli}

Stimuli for each of the two attribute-identification problems consisted of $245 \times 8$ in. index cards, on which were mounted cutouts of geometric shapes. Each card displayed a pattern based on four, three-valued dimensions: (1) number of figures (one, two, or three), (2) size of figures (small, medium, or large), (3) color of the figures (red, blue, or yellow), and (4) shape of the figures (square, circle, or triangle). Each problem of 24 trials was divided into six blocks of 4 trials each, with a 1:1 ratio of positive-to-negative instances in each block. Each of six possible orders of positive and negative instances in blocks of 4 trials was represented in each of the two problems, thus making up the 24 trials. The arrangement of stimuli was such that enough information for problem solution existed in any four-trial block.

\section{Design and Procedure}

Four patients each were assigned to one of the eight conditions formed by the combination of three binary classifications: male or female, high- or low-WIST, and paranoid or nonparanoid. Assignment to the paranoid and nonparanoid groups was determined by scores on the paranoid $(\mathrm{Pa})$ scale of the MMPI. Nonparanoid subjects were those with $T$ scores of 65 or below on the $\mathrm{Pa}$ scale, while the paranoid subjects were those with T scores of 75 or above. Subjects with a schizophrenic index (WIST) below 25 were listed in the low-WIST group, while those with an index of greater than 25 served in the high-WIST group.

The four groups of patients defined by WIST and $\mathrm{Pa}$ classifications were examined for differences in age, years of education, number of hospitalizations, scores on the ShipleyHartford IQ scale, and daily dosages of antipsychotic medication, in chlorpromazine equivalents (Byck, 1975). No differences among the four groups of patients were found for any of these characteristics by analyses of variance (all Fs $<1$ ).

Subjects were tested individually in two sessions. During the first, the Shipley-Hartford scale, the MMPI, and the WIST were administered. In the second session, each subject was given two attribute-identification problems, each based on a conjunctive relationship between two stimulus attributes.

After the stimuli were described and the general form of problem solution was explained to the subject, the first problem was presented. The subject was shown a card, which was described verbally by the experimenter. He or she was then asked if the card was a positive instance (having both relevant attributes) or a negative instance (having only one or neither of the relevant attributes) of the concept to be identified. After answering with a "positive" or a "negative," he or she was informed whether that answer was correct. The subject was then asked to state which attributes he or she believed were the critical ones. No feedback was given for this response; the next card was then presented and the process was repeated. The cards were presented one at a time until one of two criteria was met: 10 consecutive correct category responses ("positive" or "negative"), or four consecutive correct identifications of the relevant attributes. If all 24 cards were presented without either criterion being met, presentation of the cards was repeated. The second problem followed in the same manner, but had different relevant attributes.

In one of the problems, each card was removed from sight immediately after the subject's hypothesis of the relevant attributes was stated (internal memory condition), while in the other, the four cards immediately preceding each card presented on any trial remained in view, with all positive instances of the concept so marked (external memory condition). Each subject received one problem of each kind, with the order of presentation counterbalanced across subjects.

\section{RESULTS}

Patients' error scores and trials to criterion were submitted to 2 by 2 by 2 by 2 mixed analyses of variance. Sex and the WIST and paranoid dimensions were between-subjects factors, and memory condition was the within-subjects factor. A matrix of correlation coefficients is presented to provide information on the relations of independent, background, and dependent variables (Table 1). Since the two dependent variables (errors and trials to criterion) are highly correlated $[\mathrm{r}(30)=.93, \mathrm{p}<.01]$, only data on errors are reported. Figure 1 shows the mean errors $( \pm 1 \mathrm{SE})$ made by each group.

The main effect of WIST index was significant $[F(1,24)=10.52, \quad p<.01]$, as was the interaction of the WIST and $\mathrm{Pa}$ dimensions $[\mathrm{F}(1,24)=6.14$, $\mathrm{p}<.05$ ]. A test for simple main effects (collapsed across sex) showed that, within the low-WIST group, nonparanoid and paranoid subjects did not differ from each other $[F(1,24)=1.51, p>.10]$. Within the highWIST group, however, paranoid subjects made fewer errors than nonparanoid $[F(1,24)=7.52, p<.05]$. Main effects of sex and the $\mathrm{Pa}$ dimension were not significant $[\mathrm{Fs}(1,24)=1.73$ and 2.16 , respectively, both ps $>.05]$.

Means of errors of the four groups of patients were compared to the mean of the students by Dunnett $t$ tests 
Table 1

Correlation Matrix of Background, Independent, and Dependent Variables

\begin{tabular}{|c|c|c|c|c|c|c|c|c|}
\hline Variable & 1 & 2 & 3 & 4 & 5 & 6 & 7 & 8 \\
\hline $\begin{array}{l}\text { 1. Trials } \\
\text { 2. Errors } \\
\text { 3. WIST Index } \\
\text { 4. Pa Score } \\
\text { 5. IQ } \\
\text { 6. Years in School } \\
\text { 7. Number of Hospitalizations } \\
\text { 8. Age } \\
\text { 9. Drug Dosage }\end{array}$ & $\begin{array}{r}.93 \\
.54 \\
-.11 \\
-.26 \\
.28 \\
.04 \\
.01 \\
.02\end{array}$ & $\begin{array}{r}.50 \\
-.13 \\
-.17 \\
.32 \\
.01 \\
.04 \\
.22\end{array}$ & $\begin{array}{r}-.13 \\
-.39 \\
-.14 \\
.30 \\
.04 \\
-.06\end{array}$ & $\begin{array}{r}-.03 \\
.11 \\
-.02 \\
.03 \\
-.08\end{array}$ & $\begin{array}{r}.57 \\
-.13 \\
-.02 \\
.25\end{array}$ & $\begin{array}{r}.00 \\
-.09 \\
.16\end{array}$ & $\begin{array}{r}.69 \\
-.22\end{array}$ & -.09 \\
\hline
\end{tabular}

(Winer, 1971). The patients of the high-WIST nonparanoid group made significantly more errors than the students $[\mathrm{t}(30)=4.75, \mathrm{p}<.01]$, but the means of the other groups of patients did not differ significantly from that of the students. Differences in errors under the two memory conditions did not approach significance $(\mathrm{F}<1)$.

\section{DISCUSSION}

The results of this study argue for a qualification of the relationship described by Bourne et al. (1977) between a schizophrenic thought disturbance and performance on an abstract conceptual task. In this study, a performance deficit was found only in those patients who, in addition to showing high-schizophrenic indices, obtained low scores on a measure of paranoid thinking. It is important to note that the studies by Bourne et al. (1977) and the one reported here employed different conceptual tasks: attribute-identification in the present case and rule learning in the earlier study. There is enough similarity in the processes underlying both tasks, however, to expect that these results will be reproduced in studies of rule learning.

The argument that a device such as the WIST is not an unqualified predictor of a deficit in logical abilities is consistent with the contention of Wahl and Wishner (1972) that paranoid and nonparanoid schizophrenic patients suffer two unique disorders of thinking. A formal thought disorder is evident in the nonparanoid: Thinking is disorganized and regressive. On the

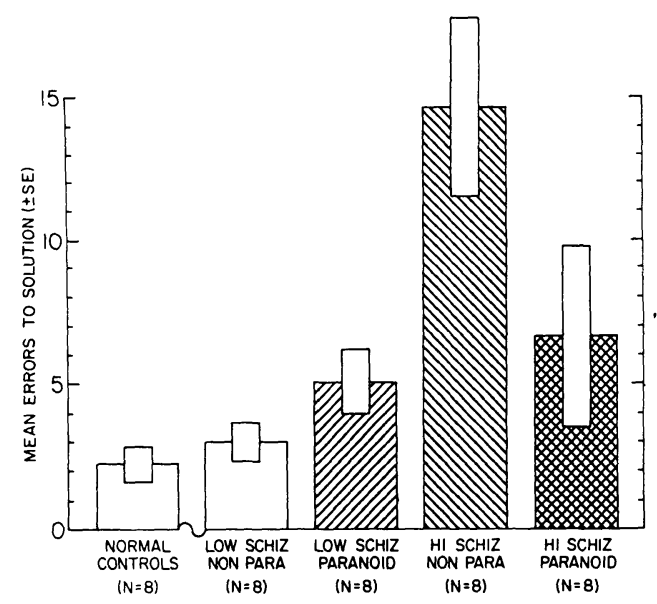

Figure 1. Mean errors on attribute-identification problems by patients and normal controls. other hand, the paranoid disorder is one of thought content, in which relatively unimpaired cognitive functioning is "misused." A primary consideration in a diagnosis of paranoid schizophrenia is the presence of a delusional system. It is within the framework of this system that the disturbance of thought takes place. Thought processes that are removed from the delusional framework may be left relatively intact. The thinking of the high-WIST paranoid subjects clearly was disturbed, as evidenced by their higher schizophrenic indices, but the disturbance may be more a reflection of an idiosyncratic content of thought than disorganization of logical thought processes per se. As the identification of conceptual attributes did not tap this disordered content, the paranoid subjects were able to perform more normally than the nonparanoid, who, with a formal thought disorder, were unable to use the necessary attentional and logical skills.

Broen (1973) has suggested that the impaired attention that is frequently seen in schizophrenia serves as a protective measure, as a means of filtering out much of the distracting stimulation that attends the early stages of schizophrenia. One way in which this filtering is thought to take place is by selective use of one sense modality. Evidence of this kind of filtering has been found in nonparanoid, but not in paranoid schizophrenics (Broen \& Nakamura, 1972). While this strategy may help the nonparanoid limit distracting stimulation, it may also lead to exclusion of relevant information. Such an attentional strategy would affect performance on an attribute-identification problem. The subject must respond verbally to information presented visually, in accordance with instructions received aurally. If the nonparanoid has limited input of information to one modality, he or she would be unable to meet the demands of problem solution, that is, attending to and comparing stimulus attributes. The paranoid subject, who does not appear to limit input of information in this way, would be able to perform more normally. An alternative to the argument for two distinct thinking disorders is the possibility that an overlay of paranoid thinking in some way overrides or compensates for the deficit associated with schizophrenia. In either case, a measure of schizophrenic thinking (such as the WIST) alone will not tell us all we need to know in order to predict logical problem solving abilities in the schizophrenic; we must also know something of this overlay of paranoid thinking.

The hypothesis that the paranoid subject would be better able than the nonparanoid to make use of the extra information provided by the external memory condition was not supported. The failure to find a difference may be a function of the relative ease of the problems used in this study. Bourne et al. (1964) found that external cues to memory have minimal effects on problems that are solved rapidly. The number of errors made by the control subjects and all of the groups of patients except the high-WIST nonparanoid group was close to the lower limit. In contrast, the high-WIST nonparanoid subjects were, in general, simply unable to solve the problems, with or without memory 
aids. It is possible that more difficult problems would make the subjects more sensitive to the memory factor.

Conceptual impairment is frequently mentioned as a symptom of schizophrenia. These data argue that, while such an impairment is indeed a correlate of schizophrenia, and one that can be predicted in part by the WIST, a full account of the impairment must also take into consideration a patient's level of paranoid thinking.

\section{REFERENCES}

BEEKER, C. G. Disjunctive and biconditional conceptual behavior in recently hospitalized schizophrenic patients. Unpublished master's thesis, University of Missouri, Kansas City, 1974.

Bourne, L. E., JR., Goldstein, S., \& Link, W. E. Concept learning as a function of availability of previously presented information. Journal of Experimental Psychology, 1964, 67, 439-448.

Bourne, L. E., Jr., Justesen, D. R., Abraham, T., Beeker, C., Brauchi, J. T., Whitaker, L. C., \& Yaroush, R. A. Limits to conceptual rule-learning by schizophrenic patients. Journal of Clinical Psychology, 1977, 33, 324-334.

BROEN, W. E., JR. Limiting the flood of stimulation: A protective deficit in chronic schizophrenia. In R. L. Solso (Ed.), Theories in cognitive psychology: The Loyola symposium. Washington, D.C: Winston, 1973.

BRoEN, W. E., JR., \& NAKamuRA, C. Y. Reduced range of sensory sensitivity in chronic nonparanoid schizophrenics. Journal of Abnormal Psychology, 1972, 79, 106-111.

Byck, R. Drugs and the treatment of psychiatric disorders. In L. S. Goodman \& A. Gillman (Eds.), The pharmacological basis of the therapeutics (5th ed.). New York: Macmillan, 1975.

Silverman, J. The problem of attention in research and theory in schizophrenia. Psychological Review, 1964, 71, 352-379.

WAHL, O., \& WISHNER, J. Schizophrenic thinking as measured by developmental tests. Journal of Nervous and Mental Disease, 1972, 155, 232-244.

WHITAKER, L. C. Whitaker index of schizophrenic thinking (WIST): Manual; Forms A and B; Scoring keys. Los Angeles: Western Psychological Services, 1973.

WINER, B. J. Statistical principles in experimental design. New York: McGraw-Hill, 1971.

YoukILIs, H. D., \& DE Wolfe, A. S. The regression hypothesis and subclassifications of schizophrenia. Journal of Abnormal Psychology, 1975, 84, 36-40.

(Received for publication November 28, 1978.) 